Ariyachandra, M.R.M.F., Samarakkody, A. and Perera, B.A.K.S., 2019. Real-virtual synchronisation: A review on the state-of-the-art geometric digital twinning of infrastructure. In: Sandanayake, Y.G., Gunatilake, S. and Waidyasekara, A. (eds). Proceedings of the $8^{\text {th }}$ World Construction Symposium, Colombo, Sri Lanka, 8-10 November 2019, pp. 502-512. DOI: doi.org/10.31705/WCS.2019.50. Available at: https://2019.ciobwcs.com/papers

\title{
REAL-VIRTUAL SYNCHRONISATION: A REVIEW ON THE STATE-OF-THE-ART GEOMETRIC DIGITAL TWINNING OF INFRASTRUCTURE
}

\author{
M.R.M.F. Ariyachandra ${ }^{1}$, Aravindi Samarakkody ${ }^{2}$ and B.A.K.S. Perera ${ }^{3}$
}

\begin{abstract}
In the United Kingdom (UK), recent developments in the construction industry have increased the demand for digitised infrastructure, which facilitates the investigation of the as-is performance of assets. This establishes the need to create and maintain up-todate digital copies of infrastructure assets, often labelled as Digital Twins. Digital twins are obtained by converting the unstructured data formats of the real-world assets, such as point clouds, into high-level digital representations. Yet, only few assets today have usable digital twins because of the high costs of the latter. This counteracts the benefits of the twins and reduces dramatically their true potential. Hence, there is a pressuring need to automate the process of creating digital twins. Geometric digital twin, the most basic form of the twin, contains only the geometry of the physical asset. This paper reviews the work done in computer vision, geometry processing, and civil engineering fields to determine the potential that exists for automatically producing geometric digital twins of infrastructure.
\end{abstract}

Keywords: Geometric Digital Twin (GDT); Infrastructure; Point Cloud Data (PCD).

\section{INTRODUCTION}

Digital twin (DT) emerged from the aerospace field and was initially defined by the National Aeronautics and Space Administration (NASA) as "an integrated multi-physics, multi-scale, probabilistic simulation of a vehicle or system that uses the best available physical models, sensor updates, fleet history, etc. to mirror the life of its flying twin" (Shafto et al., 2010). It is expected to replicate its physical asset in a digital environment. However, the data and models held in it will relate only to their intended purpose (Boschert, Heinrich and Rosen, 2018). A digital twin of an infrastructure can learn and update itself continuously using multiple data sources to represent the near real-time status and working condition of the infrastructure (HM Government, 2013). It can be maintained throughout the whole life-cycle of the infrastructure and will be continuously accessible (Parrott and Lane, 2017). Hence it can provide an early insight into the risks posed to the infrastructure by climatic events, neighbourhood structures or aging (Koch et al., 2014). According to Koch et al., (2014), there can be three types of DT in the lifecycle of an infrastructure: (a) As-designed DT produced by the design team, (b) As-built

\footnotetext{
${ }^{1}$ Department of Engineering, University of Cambridge, United Kingdom,mfa47@cam.ac.uk

${ }^{2}$ Department of Building Economics, University of Moratuwa, Sri Lanka, aravindilavanya5@gmail.com

${ }^{3}$ Department of Building Economics, University of Moratuwa, Sri Lanka, kanchan@uom.lk
} 
DT produced by the main contractor and reflecting the state of the infrastructure at the time of its completion, and (c) As-Is DT produced by the infrastructure facilities management team by surveying the infrastructure regularly. DT hereinafter unless otherwise stated specifically refers to As-Is DT, generated for the existing infrastructure.

Most recently, the Heathrow airport terminal 2B project that experienced $£ 10$ million in savings and a reduction of 5 weeks in the schedule by digitising the project (Bower, 2014). The wider adoption of DTs by 2025 for the infrastructure is expected to provide 15-25\% savings (Gerbert et al., 2016; Barbosa et al., 2017). However, West and Blackburn, (2017) claim that the generation of DTs of the existing infrastructure is highly discouraged today as the costs outweigh the benefits.

The aim of this paper is to investigate the state of the art in generating geometrically accurate models of existing infrastructure. The paper starts off by explaining the present status of digital twin generation. The subsequent sections provide a longitudinal literature review focuses on geometric modelling infrastructure. The paper finally concludes by deriving gaps in the knowledge and elaborating potential research contributions.

\section{PRESENT STATUS OF DIGITAL TWIN GENERATION}

Digital twinning is the mapping of a physical asset to a digital platform (Rossi, 2017). It can be continuously updated using data collected from sensors. The data so collected are first used to form a twin in an unstructured data format of the real-world asset, such as a point cloud data (PCD). It is a collection of XYZ coordinates of a three-dimensional (3D) coordinate system (Benli, 2015). This PCD, which is a low-level digital representation of the asset, then converted into a high-level digital representation, the DT, through a twinning phase, which structures the unstructured raw data.

Although many hardware solutions capable of collecting accurate geometrical data of infrastructure in the form of PCDs are already available, there are still only limited number of DTs of the existing infrastructure. The main reason is the difficulty experienced, even by skilled modellers, in manually generating at least a seemingly simple DT structure using PCDs. The Scan-to-BIM process (Tang et al., 2010) of creating a DT involves four major steps: (1) raw image and/or PCD capture, (2) data preparation, (3) geometric modelling and (4) semantic enrichment of the model with additional information, such as topological relationships and material specifications. The $3^{\text {rd }}$ step requires more than two thirds of the effort needed for generating the entire DT. Furthermore, the time required to manually create a DT from PCD using even the cuttingedge modelling software is almost 10 times more than that required to obtain the original point cloud (Trimble, 2017). Training of the modellers is also a necessity since DT generation is a highly domain-specific task; even proficient Computer Aided Design (CAD) professionals may not be adequately competent to manipulate modelling software without specialised training (McNell et al., 2011). Generation of a DT from PCD incurs high costs, both fixed and variable. Fixed costs involve DT modelling software license fees, cost of hardware required for using the software, and cost of training of modellers. Variable costs are the fees spent on each individual modelling project, which relate to the total number of modelling hours and the hourly labour cost. If fixed costs and the hourly labour cost remain constant, the total cost of DT generation will depend on the total number of modelling hours. Thus, cost savings can be made by using automated solutions that will reduce the total modelling time. 
The most advanced PCD-to-DT modelling software solutions are provided by major vendors, such as Autodesk, Bentley, Trimble, AVEVA and ClearEdge3D. According to Wang, Cho and Kim (2015) and Agapaki and Brilakis, (2018), although the currently available DT modelling commercial software can automate a large extent of the DT generation process, they are still far from being fully automatic. For commercial applications outside building and industrial environments, modellers have to first manually segment a PCD into subparts, and then manually fit the 3D shapes to the subparts. This demands a significant amount of time. PCDs has to be rotated repeatedly to get different views and identify the regions of interest using clipping polygons. The subsequent fitting of the accurate 3D shapes to the segmented sub-point-clusters is also challenging. Most software applications provide built-in shape libraries containing a few predefined and generic construction component primitives such as beams, walls and columns. However, the allowable primitives are also limited (Wang, Cho and Kim, 2015). Since infrastructure components usually have arbitrary shapes containing skews or imperfections, which cannot be simply fitted using idealised predefined shapes, modellers have to manually create an accurate solid form to fit each point cluster as none of the existing software packages available are capable of doing this automatically.

\section{RESEARCH ON DIGITAL TWIN GENERATION}

Automation of the modelling process can be divided into two parts: (1) object detection in PCD, and (2) 3D solid model fitting to point clusters.

\subsection{OBJECT DETECTION IN PCD}

Uijlings et al. (2012) define detection as the combination of clustering (from a PCD to point clusters) and classification (labelling the point clusters).

\subsubsection{Bottom-up Approach}

Bottom-up approach pieces together low-level primitive features like points to generate complex systems at successively higher levels until a top-level system is formed (Borenstein and Ullman, 2008). Its typical higher-level features are the surface normal (Sampath and Shan, 2010), meshes (Marton, Rusu and Beetz, 2009), surface planes/ patches (Zhang and Tang, 2015), non-uniform B-Spline surfaces (Dimitrov and Golparvar Fard, 2015; Dimitrov, Gu and Golparvar-Fard, 2016), and voxels (Vo et al., 2015). There are three main methods used in this approach.

RANdom Sample Consensus (RANSAC) has essentially two steps repeated iteratively. Firstly, hypothetical shapes are generated by randomly selecting a minimal subset of points followed by estimating the fitting model parameter of the sample subset. Secondly, the remaining points are iteratively checked to determine whether they are consistent with the model instantiated by the estimated model parameters obtained from the first step. The shape model that possesses the largest percentage of points is then extracted. TarshaKurdi, Landes and Grussenmeyer (2007) have proposed an extended RANSAC algorithm to extract roof planes from low density PCD having different complexities. Bosché (2012); Arikan et al. (2013) and Jung et al. (2014) have used RANSAC to detect planar surfaces such as walls, floors, ceilings etc. in PCD of buildings. Although this algorithm is found to be effective even in the presence of noise and outliers (Tarsha-Kurdi et al., 2007), it has its own limitations as well. Firstly, since it is used to determine different planes from a single grouping, there can often be spurious-planes (i.e. planes overlapping 
multiple reference planes or a plane snatching points from its neighbouring planes), especially around boundaries (Yan et al., 2012; Jung et al., 2014). Secondly, it requires prior knowledge about the data, which is often not the case in practice. Hence RANSACbased methods will perform well only in relatively simplified scenarios and thus will not be suitable for real infrastructure asset components whose as-weathered and as-damaged shapes would further increase the as-designed complexity.

Region Growing $(\boldsymbol{R} \boldsymbol{G})$ also has two main steps. It starts with a set of small iteratively merged areas by arbitrarily choosing initial seeds. Its second step adds in neighbouring points based on the similarity of the surface normal, curvature or co-planarity, until an edge is reached either when a non-surface point is detected or when the distance from the seed point exceeds a threshold. Xiao et al. (2013) have proposed two complementary plane segmentation algorithms: a sub-window-based RG algorithm for structured PCD, and a hybrid RG algorithm for unstructured PCD. On the other hand, Dimitrov and Golparvar Fard, (2015) have suggested an upgraded RG method through which the seed can be found adaptively. It can deal with curved surfaces within a large range of surface roughness. It excels when there are no substantive occlusions in the input PCD. Yet, this method over-segments objects when non-trivial occlusions are present. The persistent occlusions in real PCD were addressed by Xiong et al. (2013) through a learningparadigm that can detect occluded planar surfaces and estimate their shapes in building PCD. This learning paradigm cannot be applied to infrastructure because occluded surfaces in infrastructure PCD do not follow a specific pattern unlike those in a building PCD. Assuming that there are many identical rectangular openings on a wall, it detects the rectangular-shaped openings, such as windows and doorways. Similarly, Laefer and Truong-hong (2017) have developed a kernel-density-estimation-based method for modelling steel members by simulating possible occlusions. The occluded regions in infrastructure PCD do not follow repetitive patterns. Most of those occlusions, being due to on-site vegetation and long-distance scanning, are in arbitrary locations and shapes, which cannot be tackled by any of the methods. Even though, RG-based methods have been proved to be efficient at object detection in PCD, they suffer from occlusion effects, and have boundary weaknesses, as a result of the inaccurate estimation of normals or curvatures of points near region boundaries. These limitations often require manual adjustment.

Hough-Transform (HT) maps every point in the dataset to a manifold in the parameter space containing many cells that act as accumulators with each of their points casting votes. Vosselman (2009) have employed HT-based methods to detect 3D roof planes in PCD. However, Tarsha-Kurdi, Landes and Grussenmeyer (2007) have reported that plane detection rates based on HT are lower than those using RANSAC. HT is not suitable for solving higher dimensional problems since, plane detection requires a 3D Hough parameter space while cylinder detection requires a 5D Hough parameter space. Shah (2006) suggested a two-stage approach to detect cylinders in PCD to reduce computational complexity and the number of dimensions. His method transforms 5D Hough space cylinder detection problems into 2D and 3D Hough space problems. Ahmed, Haas and Haas (2014) have employed HT to detect straight cylindrical pipes using thin slices resampled from PCD. HT, however, is a powerful tool for detecting simple geometric objects within noisy and cluttered PCD. Nevertheless, it is sensitive to parameter dimensions and thus cannot be applied to shapes that have too many parameters, since then it would result in a sparse, high-dimensional accumulator that is 
poor in performance and high in memory requirements (Hassanein et al., 2015). Thus, HT is not being used for detecting infrastructure objects, which often contain skews and imperfections, because of the impossibility of describing such objects using generic shapes with limited parameters.

Hence the three main bottom-up methods RANSAC, RG, and HT, can be considered as being generally reliable for detecting generic 2D and 3D object shapes in the presence of noise and outliers. Nevertheless, their high computational requirements render them ineffective for detecting complex objects in real infrastructure PCDs, which usually contain complex geometries.

\subsubsection{Top-down Detection}

The top-down approach begins with a broad view of the picture and is broken subsequently into compositional sub-problems that are easier to solve (Kokkinos and Yuille, 2006). A pioneering study that used the top-down modelling approach is REFAB (Reverse Engineering FeAture-Based) (Thompson et al., 1999), which uses geometric constraints, such as parallelism, concentricity, perpendicularity and symmetry, to convert points to mechanical solid models. Nüchter and Hertzberg (2008) have used a relationship reasoning network for semantic mapping. They used a set of pair-wise relationship rules such as parallel, equal height, above, under, and orthogonal to coarsely classify major planes (wall, ceiling, floor and door) in an indoor PCD. The method presented by Su, Bethel and $\mathrm{Hu}$ (2016) uses a set of connectivity criteria such as proximity, orientation, and curvature, to merge and label industrial components (pipe, vessels, and walls) across voxels. In contrast, Perez-Gallardo et al. (2017) have suggested a semantic model-based system to detect the four object classes (pipes, planes, elbows, and valves) in industrial environments using topological information while Laefer and Truong-hong (2017) have leveraged a steel standard library to identify and match the cross-sections of steel frames in PCD. Recent research has relied on As-Designed documents to present the top-down modelling approach, which can simplify PCD clustering and classification tasks (Liu, Eybpoosh and Akinci, 2012). It is because the information gleaned from prior data can shift the focus from detecting objects to matching between a PCD and the existing models (Bosché, 2010). Belsky et al. (2014) have encapsulated domain expert knowledge in the form of rule sets to infer and enrich semantics for a geometric building model.

However, all these methods have been tailored for buildings, indoor environments, and industrial objects and not for infrastructure settings, as the geometric properties of infrastructure components are quite different from those of the objects found in buildings. Also, there are either few or no As-Built or As-Is models for the existing infrastructure indicating that there is only little knowledge available about embedded objects in related PCDs. The following Figure 1 represents a framework which synthesizes all object detection methods used in the studies being presented. 


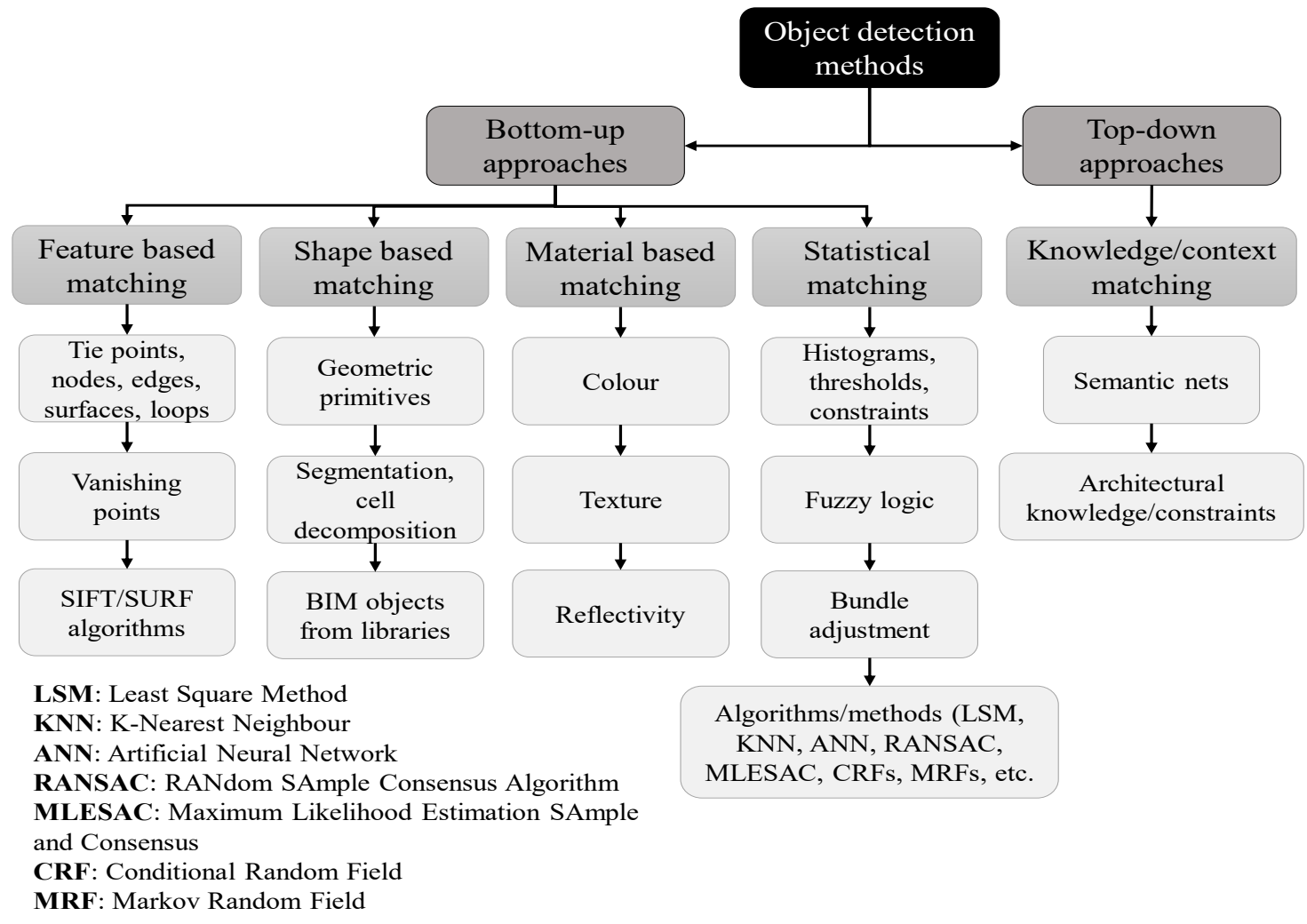

Figure 1: Object detection methods (Adopted from Volk, Stengel and Schultmann, 2014)

\subsection{Fitting the Model to a Point Cluster}

Point cluster, which is the output of the object detection step, is a subpart of the PCD. Fitting a 3D solid model to a labelled point cluster will transform a point cluster of unordered spatial points into a structured, information-rich 3D representation. The choice of digital representation depends on; (1) the nature of the object being modelled, (2) modelling technique used, and (3) application scenario where the object is brought to life. Shape representation methods can be categorized into three groups.

Implicit representation is a solid modelling approach that represents $3 \mathrm{D}$ shapes using mathematical formulations, i.e., implicit functions. Using computer-aided geometric modelling, an arbitrary constructive solid can be defined as a single real-valued function $(x, y, z))$ of three variables with $f(x, y, z) \geq 0$ (Rvachev, 1982) and for the surface, $(x, y, z)$ $=0$ (Ricci, 1973). Common implicit surface definitions include, but are not limited to, planes, spheres and ellipsoids. Implicit surfaces have difficulty in describing sharp features such as edges and vertices, although they are good at checking whether a point lies inside, outside, or on the surface (Song and Juttler, 2009). Since only a very limited number of primitives can be exactly represented by algebraic formulas, their usefulness is limited when modelling real-world 3D objects with non-idealised shapes, such as infrastructure components. In addition, infrastructure components contain defects that reduce further the effectiveness of the implicit representations.

Boundary representation (B-Rep) describes a shape using its boundary surfaces. The model formed using B-Rep is, therefore, an explicit representation, as the object is represented by a complicated data structure giving information about each of its vertices, edges, and loops and the way of joining them to form the object. The geometry of a vertex 
is given by its $(x, y)$ coordinates. The edges are straight or curved lines. A face is represented by a description of its surface with algebraic or parametric forms. Kwon et al. (2004) assuming that a construction site consists of these primitives, have introduced a rapid and accurate local spatial modelling algorithm to fit sparse PCD to planes, cuboids, and cylinders in B-Rep. Valero and Cerrada (2012) have developed methods to yield B-Rep models for indoor planar objects such as walls, ceilings and floors with high precision.

Constructive solid geometry (CSG) is a high-level volumetric representation that works both as a shape representation and as a record of the way an object has been built up (Deng, Cheng and Anumba, 2016). The final shape of the object can be represented as a combination of a set of elementary solid primitives that follow a certain type of logic. The primitives can be cuboids, cylinders, spheres, cones etc. The methods proposed by Shah (2006) and Patil et al. (2017) can be used for modelling a cylindrical piping system using simple primitives. Alternatively, the random sampling method of Schnabel, Wahl and Klein (2007) can be used to automatically model objects composed of five basic shapes (planes, spheres, cylinders, cones, and tori), which can provide a representation consisting solely of shape proxies. Walsh et al. (2013) have developed a shape library containing a generic description of objects (i.e. cuboid, cylinder) to fit point clusters using surface fitting in the form of least squares. The reliability of the method is unclear as a quantitative assessment of the fitting performance is not given in the work. Xiao and Furukawa (2012) have introduced the inverse CSG algorithm to reconstruct large-scale indoor environments with a CSG representation consisting of volumetric primitives by imposing regularization constraints. It uses only cuboids as volumetric primitives considering that they are the most common shapes found in indoor walls. The expansion of the set of geometric primitive types would, however, be necessary as construction elements can contain other shapes.

\section{GAPS IN KNOWLEDGE}

The existing object detection methods used in DT-related work concentrate mostly on a low-level of primitive generation (Dimitrov, Gu and Golparvar-Fard, 2016), i.e. surface clustering from points. Only few methods enable the direct generation of labelled point clusters. Furthermore, the existing methods focus on generating either building or industry elements, which basically take generic shapes, such as cuboids and cylinders (Patil et al., 2017), or standardized steel beams (Laefer and Truong-hong, 2017). These methods cannot be directly used to detect infrastructure components in PCD because idealised shapes are rare in real infrastructure elements. In addition, real PCD are noisy and imperfect and suffer from occlusions and sparseness. These factors render methods designed for synthetic data or simplified scenarios ineffective for infrastructure. The few DT related studies on the existing infrastructure, also have restrictive constraints; they take an idealised infrastructure solid model as the input to infer the semantic meaning of components train a classifier with generic shapes or test against a synthetic infrastructure point cloud (Zhang, Vela and Brilakis, 2014). These gaps in knowledge derives potential research contributions in the area such as;

1. Developing a method to automatically detect infrastructure structural components in PCDs - These structural components might include decks, beams for a bridge, rails, sleepers, track bed for a railway and so forth. The approach will differ with the non-idealised shape of the infrastructure component. In addition, this approach 
can combine the strengths of the data-driven strategy scenarios with very high point densities and model-based strategy in scenarios with very low point densities.

2. Developing a method to automatically fit 3D solid models to the point clusters of infrastructure components - To tackle the need of the common format, the output 3D model shall compatible with many software available, such as Industry Foundation Class (IFC) format.

3. Leveraging a reasonable 3D model assessment metrics to assess the generated GDTs of infrastructure - This is necessary as the problem of evaluating the quality and degree of automation of a generated GDT compared to its PCD has yet to be studied in depth. This assessment must compatible with the end user requirements and the level of the detail expected from the resulting model.

\section{SUMMARY AND DISCUSSIONS}

The use of DTs for existing infrastructure is limited as the perceived benefits outweigh the cost of and the effort required for DT modelling. The average time required to manually create an infrastructure GDT from a PCD using cutting edge modelling software (i.e. Autodesk Revit 2016) is about 10 times more than the time required to obtain the PCD, as the current software packages are not fully automatic. This stresses the need for automating the PCD-to-GDT process.

The knowledge gaps in the DT generation of infrastructure were identified by thoroughly reviewing in two parts the past studies on the state-of-the-art DT technologies corresponding to the two major steps of the PCD-to-GDT process: object detection in PCDs, and model fitting to point clusters. The object detection methods presently available concentrate on clustering low-level primitives to constitute surfaces and deducing the semantic meaning of the elements using high-level knowledge. However, those methods cannot effectively tackle the persistent occlusions and the varying point density problems of real PCD. In addition, none of them can address the challenges of complex geometry and topology of real infrastructure. Moreover, the existing model fitting methods concentrate on fitting idealised primitives, such as cuboids and cylinders, to indoor planar and industrial elements.

Thus, none of the existing methods can be used for detecting infrastructure components in real PCD (non-perfect data), and for modelling components with complex geometries. The contributions made by this paper to knowledge highlight the knowledge gaps and potential research contributions mentioned in Section 4, which can be used in the future to identify potential research objectives and formulate research questions.

\section{REFERENCES}

Agapaki, E. and Brilakis, I., 2018, June. State-of-practice on as-is modelling of industrial facilities. In Workshop of the European Group for Intelligent Computing in Engineering (pp. 103-124). Springer, Cham.

Ahmed, M., Haas, C. T. and Haas, R., 2014. Automatic Detection of Cylindrical Objects in Built Facilities. Journal of Computing in Civil Engineering, 28(3), pp.1-11.

Arikan, M., Schwärzler, M., Flöry, S., Wimmer, M. and Maierhofer, S., 2013. O-snap: Optimization-based snapping for modeling architecture. ACM Transactions on Graphics (TOG), 32(1), p.6.

Barbosa, F., Woetzel, J., Mischke, J., Ribeirinho, M.J., Sridhar, M., Parsons, M., Bertram, N. and Brown, S., 2017. Reinventing construction: A route to higher productivity. McKinsey Global Institute. 
Belsky, M., Eastman, C., Sacks, R., Venugopal, M., Aram, S. and Yang, D., 2014. Interoperability for precast concrete building models. PCI Journal, 59(2).

Benli, G., 2015. Handbook of Research on Emerging Digital Tools for Architectural Surveying, Modeling , and Representation. IGI Global.

Borenstein, E. and Ullman, S., 2008. Combined top-down/bottom-up segmentation. IEEE Transactions on Pattern Analysis and Machine Intelligence, 30(12), pp.2109-2125.

Bosché, F., 2010. Automated recognition of 3D CAD model objects in laser scans and calculation of asbuilt dimensions for dimensional compliance control in construction. Advanced Engineering Informatics, 24(1), pp. 107-118.

Bosché, F., 2012. Plane-based registration of construction laser scans with 3D/4D building models. Advanced Engineering Informatics, 26(1), pp.90-102.

Boschert, S., Heinrich, C. and Rosen, R., 2018. Next Generation Digital Twin., Proceedings of TMCE 2018, (May), pp. 209-218. Available at: https://www.researchgate.net/publication/325119950_Next_Generation_Digital_Twin.

Bower, D., 2014. Heathrow T2: The Queen's terminal - A lesson in value and sustainability.

Deng, Y., Cheng, J. C. P. and Anumba, C., 2016. Mapping between BIM and 3D GIS in different levels of detail using schema mediation and instance comparison. Automation in Construction, 67, pp.1-21.

Dimitrov, A. and Golparvar Fard, M., 2015. Segmentation of building point cloud models including detailed architectural/structural features and MEP systems. Automation in Construction, 51(C), pp.3245.

Dimitrov, A., Gu, R. and Golparvar-Fard, M., 2016. Non-Uniform B-Spline Surface Fitting from Unordered 3D Point Clouds for As-Built Modeling. Computer-Aided Civil and Infrastructure Engineering, 31(7), pp.483-498.

Gerbert, P., Castagnino, S., Rothballer, C., Renz, A., Filitz, R., 2016. Digital in Engineering and Construction. The Transformative Power of Building Information Modeling. The Boston Consulting Group. Available at http://futureofconstruction.org/content/uploads/2016/09/BCGDigital-inEngineering-and-Construction-Mar-2016.pdf

Hassanein, A.S., Mohammad, S., Sameer, M. and Ragab, M.E., 2015. A Survey on Hough Transform, Theory, Techniques and Applications. IJCSI International Journal of Computer Science I, 12(2).

HM Government, 2013. Construction 2025: Industrial Strategy: Government and Industry in Partnership.

Jung, J., Hong, S., Jeong, S., Kim, S., Cho, H., Hong, S. and Heo, J., 2014. Productive modeling for development of as-built BIM of existing indoor structures. Automation in Construction, 42, pp.68-77.

Klasing, K., Althoff, D., Wollherr, D. and Buss, M., 2009, May. Comparison of surface normal estimation methods for range sensing applications. In 2009 IEEE International Conference on Robotics and Automation (pp. 3206-3211). IEEE.

Koch, C., Paal, S.G., Rashidi, A., Zhu, Z., König, M. and Brilakis, I., 2014. Achievements and challenges in machine vision-based inspection of large concrete structures. Advances in Structural Engineering, 17(3), pp.303-318.

Kokkinos, I., Maragos, P. and Yuille, A., 2006, June. Bottom-up \& top-down object detection using primal sketch features and graphical models. In 2006 IEEE Computer Society Conference on Computer Vision and Pattern Recognition (CVPR'06) (Vol. 2, pp. 1893-1900). IEEE.

Kwon, S.W., Bosche, F., Kim, C., Haas, C.T. and Liapi, K.A., 2004. Fitting range data to primitives for rapid local 3D modeling using sparse range point clouds. Automation in Construction, 13(1), pp.67-81.

Laefer, D.F. and Truong-Hong, L., 2017. Toward automatic generation of 3D steel structures for building information modelling. Automation in Construction, 74, pp.66-77.

Liu, X., Eybpoosh, M. and Akinci, B., 2012. Developing as-built building information model using construction process history captured by a laser scanner and a camera. In Construction Research Congress 2012: Construction Challenges in a Flat World (pp. 1232-1241).

Marton, Z. C., Rusu, R. B. and Beetz, M., 2009. On Fast Surface Reconstruction Methods for Large and Noisy Point Clouds. In IEEE International Conference on Robotics and Automation, pp.3218-3223.

McNell, D. Allison, H., and Black, W., 2011. Building Information Modeling [Online] Available at: www.infocomm.org/cps/rde/xbcr/infocomm.Brochure_BIM.pdf 
Nüchter, A. and Hertzberg, J., 2008. Towards semantic maps for mobile robots. Robotics and Autonomous Systems, 56(11), pp.915-926.

Parrott, A. and Warshaw, L., 2017. Industry 4.0 and the digital twin. In Deloitte University Press (pp.117).

Patil, A.K., Holi, P., Lee, S.K. and Chai, Y.H., 2017. An adaptive approach for the reconstruction and modeling of as-built 3D pipelines from point clouds. Automation in Construction, 75, pp.65-78.

Perez-Gallardo, Y., Cuadrado, J.L.L., Crespo, Á.G. and de Jesús, C.G., 2017. GEODIM: a semantic modelbased system for 3D recognition of industrial scenes. In Current Trends on Knowledge-Based Systems (pp. 137-159). Springer, Cham.

Ricci, A., 1973. A constructive geometry for computer graphics. The Computer Journal, 16(2), pp.157160.

Rossi, B., 2017. Digital twinning explained. Available at: https://www.raconteur.net/businessinnovation/digital-twinning-explained.

Rvachev, V. L., 1982. Theory of R-functions and some applications. Naukova Dumka, Kiev.

Sampath, A. and Shan, J., 2009. Segmentation and reconstruction of polyhedral building roofs from aerial lidar point clouds. IEEE Transactions on Geoscience and Remote Sensing, 48(3), pp.1554-1567.

Schnabel, R., Wahl, R. and Klein, R., 2007, June. Efficient RANSAC for point-cloud shape detection. In Computer graphics forum (Vol. 26, No. 2, pp. 214-226). Oxford, UK: Blackwell Publishing Ltd.

Shafto, M., Conroy, M., Doyle, R. and Glaessgen, E., 2010. DRAFT Modeling, Simulation, information Technology \& Processing Roadmap, Technology Area. Available at: http://www.nasa.gov/pdf/501321main_TA11-MSITP-DRAFT-Nov2010-A1.pdf.

Shah, T. R., 2006. Automatic Reconstruction of Industrial Installations Using Point Clouds and Images, Publications on Geodesy 62 NCG, (May).

Song, X. and Juttler, B., 2009. Modeling and 3D Object Reconstruction by Implicitly Defined Surfaces with Sharp Features. Computers \& Graphics, 33(3), pp.321-330.

Su, Y., Bethel, J. and Hu, S., 2016. Octree-based segmentation for terrestrial LiDAR point cloud data in industrial applications. ISPRS Journal of Photogrammetry and Remote Sensing, 113, pp.59-61.

Tang, P., Huber, D., Akinci, B., Lipman, R. and Lytle, A., 2010. Automatic reconstruction of as-built building information models from laser-scanned point clouds: A review of related techniques. Automation in Construction, 19(7), pp.829-843.

Tarsha-Kurdi, F., Landes, T., Grussenmeyer, P. and Koehl, M., 2007, September. Model-driven and datadriven approaches using LIDAR data: Analysis and comparison. In ISPRS Workshop, Photogrammetric Image Analysis (PIA07) (pp. 87-92).

Tarsha-Kurdi, F., Landes, T. and Grussenmeyer, P., 2007. Hough-transform and extended ransac algorithms for automatic detection of 3D building roof planes from lidar data', IAPRS, XXXVI(1), pp. 407-412.

Thompson, W.B., Owen, J.C., Germain, H.D.S., Stark, S.R. and Henderson, T.C., 1999. Feature-based reverse engineering of mechanical parts. IEEE Transactions on Robotics and Automation, 15(1), pp.5766.

Trimble, 2017. Creating a 3D CAD Model From a laser Scanned Point Cloud (Overview).

Uijlings, J.R., Van De Sande, K.E., Gevers, T. and Smeulders, A.W., 2013. Selective search for object recognition. International Journal of Computer Vision, 104(2), pp.154-171.

Valero, E. and Cerrada, C., 2012. Automatic Method for Building Indoor Boundary Models from Dense Point Clouds Collected by Laser Scanners, Sensors, 12, pp.16099-16115.

Vo, A.V., Truong-Hong, L., Laefer, D.F. and Bertolotto, M., 2015. Octree-based region growing for point cloud segmentation. ISPRS Journal of Photogrammetry and Remote Sensing, 104, pp.88-100.

Volk, R., Stengel, J. and Schultmann, F., 2014. Automation in Construction Building Information Modeling ( BIM ) for existing buildings - Literature review and future needs. Automation in Construction, 38, pp.109-127.

Vosselman, G., 2009. Advanced point cloud processing. In Photogrammetric Week'09 (pp. 137-146). Wichmann. 
Walsh, S.B., Borello, D.J., Guldur, B. and Hajjar, J.F., 2013. Data processing of point clouds for object detection for structural engineering applications. Computer-Aided Civil and Infrastructure Engineering, 28(7), pp.495-508.

Wang, C., Cho, Y. K. and Kim, C., 2015. Automatic BIM component extraction from point clouds of existing buildings for sustainability applications. Automation in Construction, 56, pp.1-13.

West, T. D. and Blackburn, M., 2017. Is Digital Thread / Digital Twin Affordable? A Systemic Assessment of the Cost of DoD's Latest Manhattan Project. Procedia Computer Science, 114, pp.47-56.

Xiao, J., Zhang, J., Adler, B., Zhang, H. and Zhang, J., 2013. Three-dimensional point cloud plane segmentation in both structured and unstructured environments. Robotics and Autonomous Systems, 61(12), pp.1641-1652.

Xiao, J. and Furukawa, Y., 2012. Reconstructing the World's Museums. International Journal of Computer Vision, 110(3), pp.668-681.

Xiong, X., Adan, A., Akinci, B. and Huber, D., 2013. Automatic creation of semantically rich 3D building models from laser scanner data. Automation in Construction, 31, pp.325-337.

Yan, J., Jiang, W. and Shan, J., 2012. Quality analysis on ransac-based roof facets extraction from airborne lidar data. International Archives of the Photogrammetry, Remote Sensing and Spatial Information Sciences, 1, pp.367-372.

Yue, K., Huber, D., Akinci, B. and Krishnamurti, R., 2006, June. The ASDMCon project: The challenge of detecting defects on construction sites. In Third International Symposium on 3D Data Processing, Visualization, and Transmission (3DPVT'06) (pp.1048-1055). IEEE.

Zhang, C. and Tang, P., 2015. Visual complexity analysis of sparse imageries for automatic laser scan planning in dynamic environments. In Computing in Civil Engineering 2015 (pp. 271-279).

Zhang, G., Vela, P. A. and Brilakis, I., 2014. Computing in Civil and Building Engineering, The Sixth Annual International Conference on Computing in Civil and Building Engineering, pp.455-462. 